\title{
Macular Rash
}

National Cancer Institute

\section{Source}

National Cancer Institute. Macular Rash. NCI Thesaurus. Code C117107.

An outbreak of small, flat, red spots on the skin. 